\title{
Aplicación de una prueba objetiva conductual de evaluación de la influencia de los otros en el rendimiento en fútbol
}

\author{
Application of a behavioral objective evidence of evaluation \\ of the influence of others in the performance in soccer
}

\section{Ampliação de uma prova objetiva de conduta de avaliação $\mathrm{da}$ influencia dos outros no rendimento do futebol}

de la Vega Marcos, R.*, Ruiz Barquín, R.*, Fuentealba Caro, M.a A.**, Ortín Montero, F. J.***

Universidad Autónoma de Madrid ${ }^{*}$, O3 Wellbeing**, Universidad de Murcia***

\section{RESUMEN}

Se presenta el desarrollo de una prueba objetiva basada en el uso de un dinamómetro manual para analizar la influencia que ejerce la presencia de otras personas significativas, como el entrenador o los compañeros, en jugadores de fútbol infantil, cadete y juvenil de la Comunidad Autónoma de Madrid. Su aplicación permite superar las limitaciones presentadas por las pruebas de autoinforme tradicionales, analizando la validez convergente que tienen los resultados obtenidos con los encontrados en la escala de "Influencia de la evaluación del rendimiento" del cuestionario de características psicológicas relacionadas con el rendimiento deportivo factorizado CPRD-f (Olmedilla, García y Martínez, 2006). Los resultados obtenidos muestran la utilidad del empleo de una técnica objetiva como la planteada en el análisis de la influencia que tienen los otros en el rendimiento individual.

Palabras clave: facilitación social, fútbol, personalidad, pruebas objetivas. ABSTRACT

An objective test, by using a hand dynamometer is presented in order to analyze the influence of significant people as the coach or the team mates in grip strength performance of young soccer players. Through this objective test was possible to overcome the methodological limitations presented by the traditional self-reported measures. The convergent validity between the dynamometer test results and the values obtained from the "Influence of performance evaluation scale" of the Psychological Characteristics related to Sport Performance factored Questionnaire CPRD-f (Olmedilla, García y Martínez, 2006) was also analyzed. The results showed the usefulness of an objective test as the hand dynamometer to analyze the influential role of significant people on individual performance.

Keywords: social facilitation, soccer, personality, objective test.

\section{RESUMO}

Apresentamos o desenvolvimento de uma prova objetiva baseada no uso de um dinamômetro manual para analisar a influencia que exerce a presença de outras pessoas significativas, como o treinador ou os companheiros nos jogadores de futebol infantis, cadetes e juniores da Comunidad Autónoma de Madrid. Sua aplicação permite superar as limitaçóes apresentadas pelas provas auto-informes tradicionais, analisando a validez convergente que tem os resultados obtidos com os encontrados na escala de "influencia da avaliação do rendimento” do questionário de características psicológicas relacionadas com o rendimento esportivo fatorizado CPRD-f (Olmedilla, García y Martínez, 2006). Os resultados obtido mostram a utilidade da aplicação técnica objetiva como a proposta na analises da influencia que tem os outros no rendimento individual.

PALAVRAS CHAVE: facilitação social, futebol, personalidade, provas objetivas.

\section{Introducción}

Ya en el trabajo clásico y pionero del profesor de la Universidad de Indiana, Norman Triplett (1998), se analiza la influencia que ejerce la presencia de otros individuos en la realización de ciertas tareas como el resultado obtenido en pruebas de contrareloj en ciclistas que salían solos o que salían en grupo, estableciendo como los ciclistas que obtenían los mejores tiempos eran los que rodaban en grupos o en parejas, respecto a los que lo hacían en solitario que empleaban un mayor tiempo. En este

Ricardo de la Vega Marcos. C/ Francisco Tomás y Valiente, 3.
Universidad Autónoma de Madrid. Ciudad Universitaria Cantoblanco.
28049. Madrid. delavegaricardo@hotmail.com
Esta investigación ha sido parcialmente financiada gracias al proyecto de
I+D+I MICINN PSI2012-27000

mismo trabajo, el propio Triplett menciona las observaciones de un aficionado al ciclismo, Turner, para el que los ciclistas que necesitaban imponer su propio ritmo de carrera tenían un mayor número de preocupaciones y aumentaban sus niveles de producción de carbónico, de lactato y de ácido úrico, lo que disminuía la capacidad contráctil muscular y, como consecuencia, disminuía también el rendimiento respecto a las veces en las que el ritmo de carrera lo imponía otro ciclista o el pelotón.

Allport (1924), basándose en este trabajo pionero, así como en resultados similares encontrados en tareas sencillas como la de rebobinar un carrete de una cańa de pescar en solitario o en presencia de otros, acuñó el término de Facilitación Social para designar que la actividad de un individuo se puede ver influida 
por la presencia de otras personas que efectúan la misma tarea. Sin embargo, muy pronto comenzó a suscitarse una airada controversia sobre los efectos beneficiosos o perjudiciales que, sobre el rendimiento en diversas tareas, podía tener la presencia de los otros, surgiendo investigaciones aplicadas a diferentes contextos, como el educativo (Cox, 1965), el comunicativo (Henningsen y Henningsen, 2003; Micheals, Blommel, Brocato, Linkous y Rowe, 1982) o en el propio contexto deportivo (Lehman y Reifman, 1987), en donde el rendimiento alcanzado en cierto tipo de tareas, lejos de verse beneficiado por la presencia de otras personas, se veía perjudicado.

En la línea de las ideas expresadas por Wilke y Knippenberg (1990), y retomadas en una excelente revisión crítica realizada por Loriente (2007), las investigaciones establecieron dos grandes tipos de estudios: la influencia de la presencia de audiencia pasiva en el rendimiento en diversas tareas, y la influencia que tiene la presencia de otra persona que realiza la misma tarea sobre el rendimiento, lo que se conoce como coacción. Ambas líneas continuaron ofreciendo resultados contradictorios por lo que, tal y como señala Myers (2004), provocó su abandono focalizando la atención en otras áreas de estudio relevantes para la psicología social.

No es hasta 1965, en la conocida publicación de Zajonc "Social Facilitation. A solution is suggested for an old unresolved social psychological problema", que se reanudan las investigaciones y se plantea una solución plausible a los resultados contradictorios hallados hasta la fecha: la presencia de espectadores facilitaría la emisión de conductas bien aprendidas y dominadas, mientras que dificultaría la adquisición otras nuevas. En las situaciones en las que las personas se enfrentan a un contexto en el que deben realizar una tarea en presencia de otros pero la tarea está bien aprendida, la activación generada facilitaría el rendimiento mientras que, por el contrario, un aumento de la activación derivaría en una elevada ansiedad en el momento en el que se debe realizar una tarea compleja en un contexto con público. En este mismo trabajo, Zajonc establece una diferenciación relevante para nuestro estudio, en tanto que plantea que una tarea sencilla sería aquélla en la que hay pocas respuestas competitivas o alternativas que ofrecer, mientras que en una tarea compleja muchas respuestas entrarían en competición.

En la actualidad, son dos las teorías dominantes las que tratan de explicar el efecto que ejerce la presencia de los otros sobre el rendimiento individual: la teoría de la aprehensión de la evaluación y la teoría de la distracción-conflicto. En la primera (Baron y Byrne, 1998), se defiende que cuando los sujetos saben que su desempeño será evaluado y observado por los demás, se produce un contexto que deriva en la aprehensión evaluativa o percepción de la persona de estar siendo evaluado, lo que influye en la conducta resultante y, como consecuencia, en el resultado obtenido. La teoría de la distracción-conflicto (Baron, 1986), indica que la presencia ajena puede afectar al rendimiento porque distrae a la persona de la propia ejecución, aumentando la probabilidad de producir un mayor porcentaje de errores y un peor aprovechamiento de los recursos propios.

Algunos de los estudios más relevantes realizados en los últimos años sobre el estudio de la influencia de los otros en el rendimiento individual, son los realizados por Bois, Lalanne y Delforge (2009), sobre cómo influye la presencia de los padres en el aumento de la ansiedad precompetitiva de sus hijos. La muestra seleccionada fue de 341 deportistas (jugadores de baloncesto y tenis), encontrando que la presencia de los padres se asocia a un aumento en la ansiedad precompetitiva en todos los casos a excepción del tenis masculino; mientras que en el caso del tenis femenino la presencia de los padres aumentaba la presión percibida por las jugadoras respecto al resto de grupos, lo que podría indicar la necesidad de profundizar en los efectos diferenciales en función de la especialidad deportiva que se esté tratando, así como de los posibles efectos diferenciales sobre el género. Por su parte, Page y Page (2010), analizan el papel que tiene la presencia del público sobre los árbitros basándose en los datos de la obtención de mejores resultados cuando se juega en casa respecto a cuando se hace fuera, encontrando dos resultados que se consideran relevantes: en primer lugar, se encuentran diferencias individuales en función de la personalidad de los árbitros y, en segundo lugar, la cantidad de gente que observa el partido tiene una influencia sobre sus conductas relacionadas con el propio arbitraje, estableciendo un efecto diferencial de la presencia del público sobre el arbitraje realizado, mostrando como algunos árbitros son más propensos a verse influido por la multitud, que otros, lo que les lleva a concluir la necesidad de realizar un mayor número de investigaciones desde la psicología social que contribuyan a analizar el efecto que tiene el árbitro en el resultado y las variables mediadoras que pueden estar influyendo.

El estudio de la posible influencia de los otros en el rendimiento deportivo, ha sido también objeto de interés por parte de la psicología de la personalidad, encontrando numerosos estudios (Apitzsch, 1994; Arbinaga y Caracuel, 2008; De la Vega, Rivera y Ruiz, 2011; Jaenes, Godoy y Román, 2009; Marrero, Martín-Albo y Núnez, 2000; Ruiz, 2005, 2008; Salvador y Oberst, 2005; Piñar et al., 2009). Graydon y Murphy (1995), en un estudio realizado con el Eysenck Personality Inventory, con una muestra seleccionada de 20 sujetos divididos en dos grupos, uno de introvertidos y otro de extrovertidos, que debían realizar una tarea de golpeo en tenis de mesa de forma aislada y con la presencia de público, encuentran que los deportistas extrovertidos obtienen mejores resultados en tareas en las que hay público observándoles, mientras que los introvertidos obtienen un mejor rendimiento en las mismas tareas cuando las realizan de forma aislada.

Por lo que respecta a las pruebas de autoinforme generadas en el contexto deportivo para evaluar la influencia que tienen los otros sobre el individuo, Gimeno, Buceta y PérezLlantada (2001) elaboran el Cuestionario de Características 
Psicológicas Relacionadas con el Rendimiento Deportivo (CPRD) adaptando y validando para poblaciones de deportistas españoles el Psychological Skills Inventory for Sports (PSIS; Mahoney, Gabriel y Perkins, 1987). Posteriormente Olmedilla, García y Martínez (2006) han factorizado el CPRD para una muestra con futbolistas, el cuestionario final (CPRD-f) $)^{1}$ consta de 4 factores y 29 ítems: autoconfianza (10 ítems), influencia de la evaluación del rendimiento (8 ítems), ansiedad (5 ítems) y concentración (6 ítems). Dentro de este cuestionario, resulta necesario destacar para los fines de esta investigación, la escala "Influencia de la Evaluación del Rendimiento", que se centra específicamente en el impacto que tienen situaciones estresantes características de la competición deportiva, en su mayoría relacionadas con el rendimiento del deportista y la evaluación del mismo por parte de otras personas o del propio deportista, así como de la evaluación del rendimiento ante errores en competición. Una puntuación alta en esta escala, indicaría que al deportista no le afectan negativamente estas situaciones (Olmedilla et al., 2006).

En la presente investigación se presenta una prueba objetiva para analizar la influencia de otras personas significativas para el deportista sobre su rendimiento. Asimismo, se pretende analizar el nivel de correlación existente entre la escala de "Influencia de la evaluación del rendimiento" del cuestionario de características psicológicas relacionadas con el rendimiento deportivo factorizado CPRD-f, y la prueba objetiva creada, de manera que se analice su validez convergente y se constituya como una alernativa práctica, objetiva y válida para el trabajo del psicólogo del deporte cuando quiere estudiar la influencia que tienen los otros significativos en el rendimiento individual sin el empleo de autoinformes.

\section{Método}

\section{Participantes}

La muestra estuvo compuesta por 177 jugadores varones de fútbol del las Escuelas de Fútbol de Aluche $(\mathrm{N}=96)$ y de San Fermín ( $\mathrm{N}=81$ ), ubicadas en la Comunidad Autónoma de Madrid y pertenecientes al conjunto de escuelas federativas bajo supervisión y respaldo técnico de la Federación de Fútbol de Madrid. El rango de edad de los participantes es de los 11 a los 19 años $(M=14.48, D T=2.17)$. Las categorías consideradas son: Categoría Infantil (12-13 años, $\mathrm{N}=73, D T=.4)$, Categoría Cadete (14-15 años, $\mathrm{N}=47, D T=.6)$ y Categoría Juvenil (16-18 años, $\mathrm{N}=57, D T=.9)$.

\section{Instrumentos}

La medida de rendimiento que se usó fue la fuerza manual. Para conocerla se utilizó un dinamómetro manual marca Takei, modelo Grip-D T.K.K. 5401. El registro de las medi- das cuando estaban solos, con los compañeros, con el entrenador o antes del partido se hizo en una tabla de Excel que posteriormente volcamos al paquete estadístico SPSS 17.0. La percepción de la influencia de personas significativas se midió con el Cuestionario de Características Psicológicas Relacionadas con el Rendimiento Deportivo Factorizado (CPRD-f) de Olmedilla et al. (2006). Se utilizó específicamente la escala de Influencia de la evaluación del rendimiento.

\section{Procedimiento}

La investigación se realizó en dos escuelas de fútbol, Aluche y San Fermín, ambas se encuentran dentro de la Comunidad Autónoma de Madrid. Se informó a sus directores y entrenadores de los objetivos de la investigación al igual que de la forma de medición y se envió una circular explicativa a los padres para solicitar su conformidad y, en su caso, firmar un consentimiento informado.

El CPRD-f, se les aplicó en el vestuario a los jugadores decada equipo. Una vez contestado, se aplicó la prueba de "tendencia a la permeabilidad" con dinamometría antes de comenzar el entrenamiento, enmascarando el objetivo para no influir en la ejecución de la propia tarea, seńalando que se iba a medir la fuerza de mano y brazo de cada cada jugador, puesto que tiene una influencia importante en los saques de banda en los que se pretende alcanzar cierta distancia. Una vez explicado, se balanceó la condición experimental mediante un procedimiento de selección aleatoria por azar, en donde cada jugador debía de coger un papel de entre varios en los que aparecían las tres condiciones manipuladas (en presencia del entrenador, en presencia de los compañeros o realización de la prueba solo). Siguiendo esta ordenación aleatoria, se iba aplicando la prueba del dinamometría.

\section{Análisis Estadísticos}

El análisis de datos ha sido realizado mediante el programa SPSS 17.0. A continuación se presentan los análisis estadísticos desarrollados en el presente estudio:

- Análisis descriptivos: Medias y desviaciones típicas, valores mínimos y máximos para las diferentes escalas utilizadas en el estudio.

- Prueba de Normalidad: Aplicación de la prueba de Komogorov-Smirnoff $(p>.05)$.

- Análisis de diferencia de medias para varias muestras independientes: Utilización de la prueba no paramétrica Kruskall-Wallis.

- Análisis de diferencia de medias para dos muestras independientes: de la prueba no paramétrica U de MannWhitney.

- Análisis correlacionales: Utilización del Coeficiente de correlación Spearman-Brown. 


\section{Resultados}

A continuación se presentan los diferentes análisis descriptivos para las siguientes variables: Edad; Escala del CPRD-f "Influencia de la Evaluación sobre el Rendimiento"; Dinamómetro en las diferentes situaciones del estudio; Descriptivos de la diferencias entre las valores obtenidos por el dinamóme- tro solo y en presencia de otros (dinamómetro solo-presencia de compañeros, dinamómetro solo-presencia del entrenador, dinamómetro solo-antes de la competición). Los resultados obtenidos en la escala de Influencia en la evaluación sobre el rendimiento del CPRD-f1 mostraron una media de 18.74 $(D T=5.48)$ con fluctuaciones entre los 5 y 32 puntos.

Tabla 1. Estadisticos descriptivos de las cuatro situaciones experimentales y de las diferencias entre los valores obtenidos por el dinamómetro solo y en presencia de otros.

\begin{tabular}{|c|c|c|c|c|c|}
\hline Situaciones Experimentales & $\mathrm{N}$ & $\begin{array}{c}\mathrm{M} \\
(\mathrm{kg})\end{array}$ & $\begin{array}{l}\text { DT } \\
(\mathrm{kg})\end{array}$ & $\begin{array}{c}\text { Mínimo } \\
(\mathrm{kg})\end{array}$ & $\begin{array}{c}\text { Máximo } \\
(\mathrm{kg})\end{array}$ \\
\hline Dinamómetro solo & \multirow[t]{8}{*}{177} & 32.72 & 9.17 & 15.27 & 62.03 \\
\hline Dinamómetro compañeros & & 33.08 & 9.33 & 13.60 & 62.67 \\
\hline Dinamómetro entrenador & & 31.75 & 9.20 & 14.13 & 63.77 \\
\hline Dinamómetro antes de partido & & 31.26 & 9.11 & 11.43 & 59.37 \\
\hline \multicolumn{5}{|c|}{ Diferencias en los valores solo y en presencia de otros } & \\
\hline Dinamómetro solo/compañeros & & -.48 & 2.84 & -12.50 & 7.13 \\
\hline Dinamómetro solo/entrenador & & 1.17 & 1.89 & -4.50 & 7.83 \\
\hline Dinamómetro solo/antes del partido & & 1.34 & 2.20 & -7.57 & 9.63 \\
\hline
\end{tabular}

Como se puede ver en la tabla 1 , cuando los futbolistas son medidos en presencia de los compañeros, las media registra$\mathrm{da}(M=33.08)$ es ligeramente mayor que en las otras tres situaciones experimentales. La medición máxima se registró cuando los futbolistas fueron medidos en presencia solo del entrenador (63.77). En la tabla 1 se muestran las diferencias de medias entre los valores obtenidos por los participantes en las tres situaciones de estudio. La diferencia entre solo y en presencia de compañeros es de $-.48(D T=2.84)$, entre solo y en presencia del entrenador es de $1.17(D T=1.89)$ y finalmente entre solo y antes del partido es de $1.34(D T=2.20)$.

Para determinar la utilización de pruebas paramétricas o no paramétricas, se desarrolla la prueba de normalidad de Kolmogorov-Smirnoff ( $p>$.05). Los resultados obtenidos muestran cómo en las cuatro situaciones experimentales donde se utiliza el dinamómetro se cumple con el supuesto de normalidad: solo $(p=.60)$, con compañeros $(p=.79)$, con entrenador $(p=.71)$ y antes del partido $(p=.76)$.

Con el fin de observar posibles diferencias en la prueba de dinamometría en función de la categoría de edad deportiva a la que pertenecen los jugadores, se realizan los análisis de diferencia de medias para varias muestras independientes mediante la prueba de Kruskall-Wallis, encontrando los siguientes valores: dinamómetro solo $\left(X_{(2)}^{2}=89.70, p<.01\right)$, dinamómetro con compañeros $\left(X_{(2)}^{2}=90.75, p<.01\right)$, dinamómetro con entrenador $\left(X_{(2)}^{2}=97.80, p<.01\right)$ y dinamómetro antes del partido $\left(X_{(2)}^{2}=90.47, \mathrm{p}<.01\right)$. Los resultados mostraron diferencias estadísticamente significativas $(p<0.01)$ en las cuatro situaciones de estudio consideradas en función de la categoría de edad deportiva, lo que responde a las expectativas iniciales sobre la influencia de la edad en la acción de fuerza ejercida con el dinamómetro.

Con el fin de observar posibles diferencias en la escala de "Influencia de la Evaluación sobre el Rendimiento" en función de la categoría de edad deportiva a la que pertenecen los deportistas, se desarrolla el análisis no paramétrico de diferencia de medias para los tres grupos (infantil, cadete y juvenil) a través de la prueba Kruskall-Wallis. Los resultaron mostraron que no existieron diferencias, es decir, que la edad no parece influir sobre la posible influencia que tienen los demás sobre el rendimiento personal evaluado mediante autoinforme $\left(X_{(2)}^{2}=1.38, p<.50\right)$.

A continuación se presenta un análisis de diferencia de medias para varias muestras independientes utilizando el análisis de diferencia de medias no paramétrico de Kruskall-Wallis, con el objetivo de establecer posibles diferencias entre los tres grupos de edad en función del diferencial "nivel de rendimiento" obtenido al restar los valores del dinamómetro en la situación de "solo" en comparación con la presencia de compañeros, entrenador y antes de la competición. Los resultados mostraron diferencias significativas en los valores del dinamómetro obtenidos en la situación de "solo" y en la "presencia del entrenador" $\left(X_{(2)}^{2}=6.18, p=.04\right)$. Asimismo, existe una tendencia a la significación en la escala "solo" en comparación a "en presencia de compañeros" $\left(X_{(2)}^{2}=5.41, p=.06\right)$. Este valor resulta muy relevante para los propios intereses de la investigación, en tanto 
que permite evidenciar diferencias que, como se ha señalado en el párrafo anterior, no aparecen cuando se emplea la escala de "Influencia en la evaluación del rendimiento" del CPRD.

Con el objetivo de poder establecer de forma precisa entre qué grupos de edad pueden existir diferencias en las escalas "dinamómetro solo/compañeros" y "dinamómetro solo/ entrenador", se desarrollan de dos en dos, un análisis de diferencia de medias para muestras independientes a través del estadístico no paramétrico U de Mann-Whitney. Para ello, realizamos los análisis diferenciales por pares: infantil-cadete, infantil-juvenil, y cadete-juvenil.

Tabla 2. Resultados de la prueba U de Mann-Whitney para los grupos infantil y cadete, infantil y juvenil, y cadete y juvenil considerando las dos condiciones de diferencial de nivel de rendimiento estadisticamente significativas en la prueba Kruskall-Wallis.

\begin{tabular}{lcccc}
\hline & \multicolumn{2}{c}{ Dinamómetro } & \multicolumn{2}{c}{ Dinamómetro } \\
Grupos de Edad & $\mathrm{Z}$ & $\mathrm{p}$ & $\mathrm{Z}$ & $\mathrm{p}$ \\
\hline Infantil - Cadete & -2.20 & $.02^{*}$ & -.37 & .70 \\
Infantil - Juvenil & -.39 & .69 & -2.47 & $.01^{*}$ \\
Cadete - Juvenil & -1.83 & $.06^{\dagger}$ & -1.64 & .10 \\
\hline
\end{tabular}

En la comparación de los grupos infantil y cadete, los resultados muestran diferencias estadísticamente significativas ( $p$ $<.05)$ sólo en la condición "Dinamómetro solo/compañeros”, obteniéndose mayores niveles en el grupo Infantil. Al tomar como referencia el valor diferencial, se considera que una puntuación mayor refleja una mayor variabilidad en la respuesta de fuerza ejercida y, por lo tanto, la interpretación debe realizarse sobre que a la categoría infantil le influye más que a la cadete la presencia de los compañeros. Respecto a la comparación infantil-juvenil, los resultados muestran di- ferencias estadísticamente significativas $(p<.05)$ en la condición "Dinamómetro solo/entrenador", obteniéndose mayores niveles en el grupo infantil; al igual que se señaló en el anterior resultado, la interpretación de este contraste hace referencia a que a la categoria infantil, le influye más la presencia del entrenador que a la categoría juvenil. Por último, al comparar el grupo cadete con el juvenil, no se muestran resultados estadísticamente significativos, si bien existe una tendencia a la significación estadística en la escala "Dinamómetro solo/ compañeros" $(p<.10)$.

Tabla 3.- Análisis correlacional a través del Coeficiente de correlación de Spearman para el establecimiento de posibles relaciones entre la escala de Influencia de la Evaluación sobre el rendimiento y las tres situaciones de diferencial de rendimiento

\begin{tabular}{|c|c|c|c|c|}
\hline & $\begin{array}{l}\text { Influencia de la } \\
\text { Evaluación sobre el } \\
\text { Rendimiento }\end{array}$ & $\begin{array}{l}\text { Dinamómetro } \\
\text { Solo/compañeros }\end{array}$ & $\begin{array}{l}\text { Dinamómetro } \\
\text { Solo/entrenador }\end{array}$ & $\begin{array}{c}\text { Dinamómetro } \\
\text { solo/antes del } \\
\text { partido }\end{array}$ \\
\hline $\begin{array}{l}\text { Influencia de la Evaluación sobre el } \\
\text { Rendimiento }\end{array}$ & 1,00 &,- 035 &,$- 22^{* *}$ &,$- 17^{*}$ \\
\hline $\begin{array}{l}\text { Dinamómetro } \\
\text { Solo/compañeros }\end{array}$ & & 1,00 &, $48^{* *}$ &, $40^{* *}$ \\
\hline $\begin{array}{l}\text { Dinamómetro } \\
\text { Solo/ entrenador }\end{array}$ & & & 1,00 &, $40^{* *}$ \\
\hline $\begin{array}{l}\text { Dinamómetro } \\
\text { Solo/antes del partido }\end{array}$ & & & & 1,00 \\
\hline
\end{tabular}

${ }^{*} p<.05 ;{ }^{* *} \mathrm{p}<.01$

Las resultados muestran una correlación significativa entre la escala de Influencia sobre la Evaluación del Rendimiento y las puntuaciones diferenciales entre la situación solo y en presencia del entrenador $(p<.01)$. La interpretación de este resultado debe hacerse valorando que en esta escala varios de los ítems que la componen hacen referencia, precisamente, a la posible influencia del técnico sobre el rendimiento del deportista, lo que nos permite valorar la validez conver- 
gente pretendida en el estudio planteado. Por otra parte, los resultados mostraron que la escala sobre la Influencia de la Evaluación sobre el Rendimiento y la diferencia en los valores del dinamómetro en las situaciones solo y antes del partido fueron independientes, lo que puede interpretarse también porque en la escala del CPRD no se hace referencia al contexto precompetitivo en la influencia del rendimiento alcanzado $(p>.05)$.

\section{Discusión}

Se puede afirmar que la prueba empleada para evaluar la influencia que tienen los demás en rendimiento individual, posee un gran valor por su carácter objetivo y porque, como queda reflejado en la investigación, se encuentran diferencias en el rendimiento alcanzado por los jugadores en función del momento en el que se les solicita y en función de la posible influencia que los agentes más influyentes para ellos pueden ejercer sobre su rendimiento en, al menos, varias de las comparaciones que se han planteado.

Los resultados encontrados demuestran que la prueba utilizada cumple con los parámetros establecidos por diversos autores como Catell y Warburton (1976), Ribes (1990), Santacreu et al. (2002); quienes mencionan que la evaluación debe realizarse mediante pruebas conductuales, prácticas y objetivas que permitan obtener resultados de forma simple. La evaluación mediante dinamometría, permite a su vez no tener que incluir habilidades específicas que puedan influir sobre los resultados encontrados, de éste modo seguimos la pauta establecido por Hernández, Santacreu y Rubio (1999).

Como prueba objetiva es un buen complemento para un cuestionario de autoinforme como el CPRD-f pues queda de manifiesto a partir de los resultados diferenciales obtenidos que las personas que mayores puntuaciones obtuvieron en la escala de Influencia de la Evaluación del Rendimiento del CPRD-f, son las que en la prueba mostraron menos variación en las puntuaciones en función de las comparaciones por categoría realizadas lo que nos muestra congruencia entre ambas pruebas.

Por lo que respecta a profundizar acerca de en qué categorías existe una mayor influencia de los otros sobre el rendimiento individual, la categoría infantil es la que se muestra más afectada respecta a las comparaciones con los otros dos grupos, cadetes y juveniles, si bien en éste último sólo se encuentra una tendencia a la significación. Estos resultados enfatizan la importancia que debe tener el cuidado en el fútbol base y formativo sobre los estilos de liderazgo ejercidos por los técnicos y por el cuidado exquisito que se debe tener hacia los jugadores de estas edades. En la edad adolescente es donde más influyen los demás en el rendimiento y este factor debería provocar el énfasis, por parte de las escuelas de técnicos deportivos, del desarrollo adecuado de metodologías que permitan comprender a los técnicos la realidad.

Respecto a la contrastación de la utilidad de los dos principales marcos teóricos mencionados para explicar el efecto demostrado de la presencia de los otros sobre el rendimiento, la teoría de la aprehensión de la evaluación y la teoría de la distracción-conflicto, nuestros resultados no permiten establecer resultados concluyentes, si bien se considera que resulta más plausible explicar la alteración del rendimiento mediante el empleo de una prueba tan simple como la de dinamometría por la teoría de la aprehensión evaluativa de Baron y Byrne, (1998), en tanto que defiende que la consecuencia de saberse evaluados en el rendimiento produce un sentimiento de reactividad negativa como el encontrado, al menos parcialmente. Por lo que respecta a la teoría de la distracción-conflicto, la nula habilidad técnica y sencillez de ejecución implicada en la prueba de dinamometría, permite aislar el componente perceptivo y decisional, que se encuentra inmerso en muchas tareas motrices complejas, a su mínima expresión, dependiendo el resultado del componente ejecutivo controlando, de este modo, que la propia naturaleza de las habildiades implicadas permitan explicar los resultados.

En futuras investigaciones se debe seguir profundizando con detenimiento en el empleo de técnicas de evaluación como la empleada en este estudio, así como en las características concretas que afectan a los procesos de influencia intra e intergrupales en el deporte y en los efectos diferenciales que, por ejemplo, tienen los procesos de influencia de los otros sobre el género, la edad y la categoría profesional de los jugadores. Resulta interesante, por tanto, la posibilidad que ofrece este estudio como punto de partida para la posible evaluación, mediante una prueba objetiva y simple como es ésta, de la medida en la que el rendimiento de un deportista se puede ver influido por los demás, permitiendo estructurar un trabajo más adecuado a los entrenadores y estructurar, a su vez, contexto de práctica que permitan optimizar los recursos de los propios deportista en los diferentes rangos de edad.

Por último, como limitaciones del trabajo, se plantea la necesidad de ampliar la muestra a otros deportes, así como estudiar los posibles efectos diferenciales que puede tener la presencia de los otros en función del género del deportista y de su nivel de experiencia, pudiendo extrapolar los resultados del deporte de base, como es nuestro caso, al deporte de alta competición.

\section{Referencias bibliográficas}


de Psicología del Deporte, 3 (2), 89-99.

3. Arbinaga, F. y Caracuel, J.C. (2008). Rasgos de personalidad en fisicoculturistas y relaciones con variables antropométricas y conductas deportivas. Revista de Psicología del Deporte, 17 (1), 43-59.

4. Baron, R.A. y Byrne, D. (1998). Psicología Social. Madrid: Prentice Hall.

5. Baron, R.S. (1986). Distraction-conflict theory. Progress and problems. Advances in Experimental Social Psychology, 19, 1-40.

6. Bois, J., Lalanne, J. y Delforge, C. (2009). The influence of parenting practices and parental presence on children's and adolescents' pre-competitive anxiety. Journal of Sports Sciences, 27(10), 995-1005.

7. Catell, R.B. y Barbuton, E.W. (1976). Objective personality and motivation test. Urbana, Champing, III: University of Illinois Press.

8. Cox, F.N. (1965). Some relationships between test anxiety, presence or absence of male persons, and boys' performance on a repetitive motor task. Journal of Experimental Child Psychology, 6, 100-112.

9. De la Vega, R., Rivera, O. y Ruiz, R. (2011). Personalidad resistente y carreras de fondo: comparativa entre ultra fondo y diez kilómetros. Revista de Psicología del Deporte, 20(2), 445-454.

10. Gimeno, F., Buceta J.M. y Pérez-Llanta, M. (2001). El cuestionario "Características Psicológicas Relacionadas con el Rendimiento Deportivo” (CPRD): Características psicométricas. Análise Psicológica, 1 (XIX), 93-113.

11. Henningsen, D.D. y Henningsen, M.L.M. (2003). Examining social influence in information sharing contexts. Small Group Research, 34, 391-412.

12. Hernández, J.M., Santacreu, J. y Rubio, V. (1999). Evaluación de la personalidad: una alternativa teórico- metodológica. Escritos de Psicología, 3, 20-28.

13. Jaenes, J.C., Godoy, D. y Román, F.M. (2009). Personalidad resistente en maratonianos: un estudio sobre el control, compromiso y desafío de corredoras y corredores de maratón. Revista de Psicología del Deporte, 18 (2), 217-234,

14. Mahoney, M.J., Gabriel, T.J. y Perkins, T.S. (1987). Psychological skills and exceptional athletic performance. The Sport Psychologist, 1, 181-199.

15. Marrero, G., Martín-Albo, J. y Núnez, J.L. (2000). Perfil de personalidad del tenista. Revista de Psicología del Deporte, 9(12), 21-38.

16. Micheals, J.W., Blommel, J.M., Brocato, R.M., Linkous, R.A. y Rowe, J.S. (1982). Social facilitation and inhibition in a natural setting. Replications in Social Psychology, 2, 21-24.

17. Myers, D. (2004). Exploraciones de la Psicología Social. Madrid: McGraw-Hill.
18. Lehman, D.R. y Reifman, A. (1987). Spectator influence on basketball officiating. The Journal of Social Psychology, 127, 673-675.

19. Loriente, C. (2007). Crítica teórica y práctica a la Teoría de la Facilitación Social. Athenea Digital, 12, 130-143

20. Olmedilla, A., García, C. y Martínez, F. (2006). Factores Psicológicos y Vulnerabilidad a las lesiones deportivas: un estudio en futbolistas. Revista de Psicología del Deporte, 15(1), 37-52.

21. Page, L. y Page. K. (2010). Alone against the crowd: Individual differences in referees' ability to cope under pressure. Journal of Economic Psychology 31, 192-199

22. Piñar, M.I, Cárdenas, D., Alarcón, F., Escobar, R, Estévez, F. y Torre, E. (2009). Características de las dimensiones y subdimensiones de la personalidad en jóvenes jugadores de baloncesto. Revista de Psicología del Deporte, 18 (3), 415-419.

23. Ribes, E. (1990). Aptitudes sustitutivas y planeación del comportamiento inteligente en instituciones educativas. En E. Ribes (Ed.) Psicología General, México: Trillas pp. 202-230.

24. Ruiz, R. (2005). Análisis de las diferencias de personalidad en el deporte del judo a nivel competitivo en función de la variable sexo y categoría de edad deportiva. Cuadernos de Psicología del Deporte, 5 (1,2), 29-48.

25. Ruiz, R. (2008). Aportaciones del análisis subdimensional del cuestionario de personalidad BFQ para la predicción del rendimiento en judokas jóvenes de competición. Cuadernos de Psicología del Deporte, 8 (1), 5-29.

26. Salvador, J. y Oberst, U. (2005). Narcisismo en practicantes de musculación y practicantes de natación, un estudio comparativo. Revista de Psicología del Deporte, 14 (1), 75-83.

27. Santacreu, J., Hernández, J., Adarraga, P., \& Márquez, M. (2002). La personalidad en el marco de una teoría del comportamiento human. Madrid: Pirámide.

28. Triplett, $N$ (1998). The dynamogenic factors in pacemaking and competition. American Journal of Psychology, 9, 507-533.

29. Wilke, H. y Van Knippenberg, A. (1990). Rendimiento de grupo. En J. P. Codol, M. Hewstone, G. M.

30. Stephenson \& W. Stroebe (Eds.), Introducción a la psicología social. Una perspectiva europea (pp. 307-339). Barcelona: Ariel.

31. Zajonc, R.B. (1965). Social facilitation, Science, 149, 269-274. Recuperado de http://faculty.babson.edu/krollaglorg_site/soc_psych/zajonc_soc_ fac.html [Abril, 2009]. 